\title{
Photoacoustic monitoring of inhomogeneous curing processes in polystyrene emulsions
}

\author{
M Vargas-Luna ${ }^{1}$, G Gutiérrez-Juárez ${ }^{1}$, J M Rodríguez-Vizcaíno ${ }^{1}$, \\ J B Varela-Nájera ${ }^{1}$, J M Rodríguez-Palencia ${ }^{1}$, \\ J Bernal-Alvarado ${ }^{1}$, M Sosa ${ }^{1}$ and $\mathbf{J} \mathbf{J}$ Alvarado-Gil ${ }^{2}$ \\ ${ }^{1}$ Instituto de Física, Universidad de Guanajuato, A.P. E-143, C.P. 37150 León, Guanajuato, \\ México \\ ${ }^{2}$ Centro de Investigación y de Estudios Avanzados del IPN, Unidad Mérida, \\ Antigua Carretera a Progreso Km. 6, A.P. 73 Cordemex, Mérida 97310, Yucatán
}

Received 18 February 2002, in final form 1 May 2002

Published 18 June 2002

Online at stacks.iop.org/JPhysD/35/1532

\begin{abstract}
The time evolution of the inhomogeneous curing process of polystyrene emulsions is studied using a variant of the conventional photoacoustic (PA) technique. The thermal effusivity, as a function of time, is determined in order to monitor the sintering process of a styrene emulsion in different steps of the manufacturing procedure. PA measurements of thermal effusivity show a sigmoidal growth as a function of time during the curing process. The parameterization of these curves permits the determination of the characteristic curing time and velocity of the process. A decreasing of the curing time and an increasing curing velocity for the final steps of the manufacturing process are observed. The feasibility of our approach and its potentiality for the characterization of other curing process are discussed.
\end{abstract}

\section{Introduction}

The determination of thermal properties is a useful tool in the study of materials and processes. In the last twenty-five years, the photoacoustic (PA) techniques have proven to be a valuable method for the thermal characterization of a wide range of solids showing, in many cases, significant advantages over traditional techniques [1]. The versatility of these techniques are based on the fact that they look directly at the heat generated in a sample, due to non-radiative de-excitation processes, following the absorption of the modulated light that impinges upon the sample. In particular, the microphone PA techniques are based on the PA effect, which consists on the sound generation produced by pressure changes in the PA gas chamber due to a modulated heating.

The PA techniques in liquids have been widely used, mainly, for the characterization of optical properties [2-4]. With respect to the thermal properties in liquids, the open PA cell has been used to measure the thermal effusivity of transparent liquid samples [5]. Thermal effusivity is a thermal property given by $\varepsilon=\sqrt{\kappa \rho c}$, where $\kappa$ is the thermal conductivity and $\rho c$ is the heat capacity per unit volume.
Thermal effusivity is the physical magnitude that quantifies the heat flux density exchange between the sample surface and its surroundings [6]; this magnitude is related with the so-called thermal impedance [7]. Recently it has been shown that the restriction of optical transparency can be surpassed if a variant of the conventional PA cell is used. In this case, the thermal effusivity can be determined for all kind of liquids [8].

On the other hand, the PA detection has been applied to study several transient phenomena, such as laser polymer ablation $[9,10]$, oxygen evolution during photosynthesis [11], and the evaporation and contraction dynamics of a droplet wetting a flat metallic surface [12].

In this paper, the potentialities of the PA techniques in the monitoring of inhomogeneous curing processes are explored. Special interest is given to the case where the curing occurs in layered form [13]. The studied system, consists of a water based polystyrene emulsion [14], deposited on a metallic substrate, in such a way that the curing process begins on the surface in contact with the air, as a consequence of water loss.

It is shown that the inhomogeneous kinetic curing process in layered form can be followed by monitoring the thermal effusivity as a function of time, using the alternative 
configuration of the conventional PA cell given by BalderasLópez et al [8]. It is also shown that the process obeys a second-order kinetics. The fit of the experimental results provides the characteristic time and velocity of this process. These results give a clue for an alternative technique for quality control and the specification of optimal periods of time in the manufacturing process.

\section{Experimental arrangement}

The PA investigation of the curing process of emulsions was carried out using the PA cell shown in figure 1(a). In this configuration the PA cell is closed, at the bottom end, by a glass window and, at the top end, by a removable substrate. An electret microphone, coupled to the cavity wall, is used to sense the pressure fluctuations in the PA chamber produced by the periodic heating of the substrate due to the pumping beam. This substrate is used as a reference material. The sample is deposited on the external surface of this reference material. The experimental arrangement consists of a $100 \mathrm{~mW} \mathrm{Ar}^{+}$laser beam, which is mechanically modulated by an optical chopper at constant frequency $(f=7 \mathrm{~Hz}$ ), for all experiments, and focused onto the reference material through fibre optic bundles. The microphone signal is fed into a lock-in amplifier, from where the output signal amplitude is recorded, as a function of time, in a personal computer. All measurements were performed using a commercial stainless steel foil of $256 \mu \mathrm{m}$ thickness as a reference material. When the thermal diffusivity $(\alpha)$ of this kind of steel was measured by the Mirage technique [15], the value was found to be $7.0 \times 10^{-6} \mathrm{~m}^{2} \mathrm{~s}^{-1}$, having, for the modulating frequency used, a thermal length of $560 \mu \mathrm{m}$, about twice the thickness of the steel foil. On the other hand, the thermal effusivity was measured by PA technique, getting $1.0 \times 10^{4} \mathrm{~W} \mathrm{~s}^{1 / 2} \mathrm{~m}^{-2} \mathrm{~K}^{-1}$.

(a)

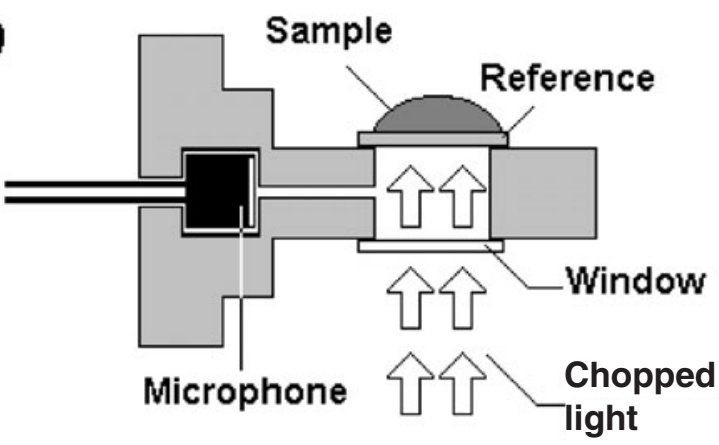

(b)

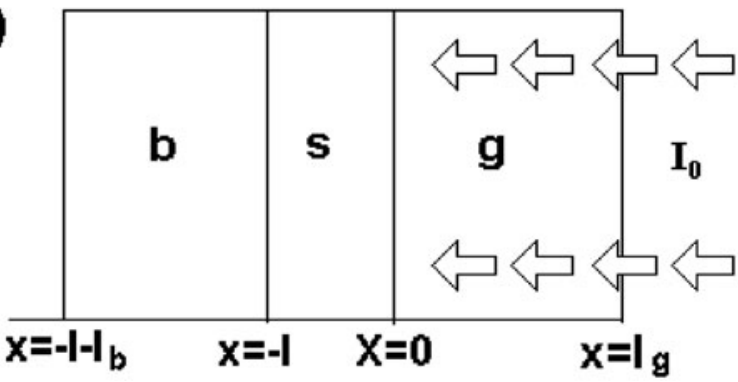

Figure 1. (a) Schematic arrangement of the conventional PA experiment. (b) Geometrical configuration of the PA cell.
The samples studied were emulsions composed by two phases, with water as the continuous phase and a mixture of several compounds as the dispersed, where the main component is polystyrene. The manufacturing process of the samples, which are controlled composition emulsions, include a $15 \mathrm{~h}$ pre-emulsion dosage cycle, in which the main change is that the polymer size inside the micelle increases in time during this cycle. These samples correspond to seven of the steps along the manufacturing procedure (pre-emulsion dosage cycle), named S1 up to S7. They were taken each $2 \mathrm{~h}$ beginning from hour 3 (step 1 sample) up to hour 15 (step 7 sample).

\section{Theory}

The geometry shown in figure $1(b)$ represents schematically the physical configuration of our experiments. A modulated laser beam, $I(t)=I_{0}(1+\cos (\omega t)) / 2$, is impinged upon the internal surface of the substrate (s), of thickness $l$, which is in contact with the air $(\mathrm{g})$ of the PA chamber. For these experiments the backing material (b), of thickness $l_{\mathrm{b}}$, is the sample to be studied.

According to the Rosencwaig and Gersho model [16], the PA signal is determined by the temperature fluctuation, $\theta$, at the air-substrate interface $(x=0)$. Solving the thermal diffusion equation for the configuration shown in figure $1(b)$, one gets

$$
\theta=\theta_{0}\left[\frac{b \tanh (\sigma l)+1}{b \operatorname{coth}(\sigma l)+1}\right],
$$

here $\sigma$ is the complex thermal diffusion coefficient, defined by $\sigma=(1+i) a$, with $a$ defined by $(\pi f / \alpha)^{1 / 2}$. Here $b=\varepsilon_{\mathrm{b}} / \varepsilon$ is the thermal coupling coefficient, with $\varepsilon$ and $\varepsilon_{\mathrm{b}}$ the thermal effusivities of the substrate and sample, respectively. $\theta_{0}$ is the expression for the temperature fluctuation in the absence of the sample on the substrate, which is given by $\theta_{0}=\left(I_{0} / 2 k \sigma\right) \operatorname{coth}(\sigma l)$. In arriving at equation (1), the fact that the effusivity of air is much smaller than the thermal effusivity of the substrate is used. Due to fact that the substrate is optically opaque, it has been considered that the optical absorption coefficient, $\beta$, is much larger than the absolute value of the complex thermal diffusion coefficient, $|\sigma|$, for the frequency used here. Moreover, the sample thickness, $l_{\mathrm{b}}$, is supposed to be much larger than its thermal diffusion length, which is given by $\mu_{\mathrm{b}}=\left(\alpha_{\mathrm{b}} / \pi f\right)^{1 / 2}$, being $\alpha_{\mathrm{b}}$ the thermal diffusivity of the sample. From equation (1) the amplitude of the ratio $\theta / \theta_{0}$ is given by

$$
\begin{aligned}
q= & \left|\frac{\theta}{\theta_{0}}\right|=\frac{\sqrt{\cosh (2 a l)-\cos (2 a l)}}{\sqrt{\cosh (2 a l)+\cos (2 a l)}} \\
& \times \frac{\sqrt{(b+1)^{2} \mathrm{e}^{2 a l}+(b-1)^{2} \mathrm{e}^{-2 a l}-2\left(b^{2}-1\right) \cos (2 a l)}}{\sqrt{(b+1)^{2} \mathrm{e}^{2 a l}+(b-1)^{2} \mathrm{e}^{-2 a l}+2\left(b^{2}-1\right) \cos (2 a l)}} .
\end{aligned}
$$

The thermal coupling coefficient $(b)$ can be obtained from solving this equation both for the phase or amplitude experimental data. From now on only the amplitude is used, given that the thermal parameters for the substrate are known and the phase analysis does not give additional information. 
Solving equation (2) for the thermal coupling coefficient, one gets the sample effusivity

$$
\varepsilon_{\mathrm{b}}=\varepsilon\left(\frac{-B+\sqrt{B^{2}-A C}}{A}\right),
$$

where

$$
\begin{gathered}
A=\left(q^{2}-1\right)\left[\cosh ^{2}(2 x)+\cos ^{2}(2 x)\right] \\
+2\left(q^{2}+1\right) \cosh (2 x) \cos (2 x), \\
B=\sinh (2 x)\left[\left(q^{2}-1\right) \cosh (2 x)+\left(q^{2}+1\right) \cos (2 x)\right], \\
C=\left(q^{2}-1\right)\left[\cosh ^{2}(2 x)-\cos ^{2}(2 x)\right]
\end{gathered}
$$

and $x=a l$.

Measuring the evolution of $q=q(t)$, the values of $\varepsilon_{\mathrm{b}}(t)$ can be obtained.

\section{Results and discussion}

In order to know the concentration of polystyrene in the emulsions, a study of the weight loss, due to the evaporation of the continuous phase, during the emulsion curing has been performed. From this experiment it can be inferred that, at the beginning of the experiment, the emulsion has a proportion of about $45 \%$ of polystyrene and $55 \%$ of continuous phase.

In figure 2 , the thermal effusivity as a function of time of two typical samples, corresponding to S1 and S7, is shown. The first part (from 0 to $400 \mathrm{~s}$ approximately) corresponds to the PA signal without sample, after that, the sample is placed above the substrate. During the time interval, from 450 to $820 \mathrm{~s}$, the signal shows a stationary behaviour and for $t>820 \mathrm{~s}$, the signal shows a rapid decay, becoming stationary again at $t>1100 \mathrm{~s}$. As can be observed in this figure, PA measurements of the thermal effusivity follow a second-order kinetics with an asymptotic behaviour at the final stage. In order to quantify the features of this behaviour, a sigmoid fitting function is proposed for the thermal effusivity of the samples:

$$
\varepsilon_{\mathrm{b}}=\varepsilon_{\mathrm{p}}+\frac{\varepsilon_{\mathrm{e}}-\varepsilon_{\mathrm{p}}}{1+\mathrm{e}^{\left(t-t_{0}\right) / t_{\mathrm{d}}}},
$$

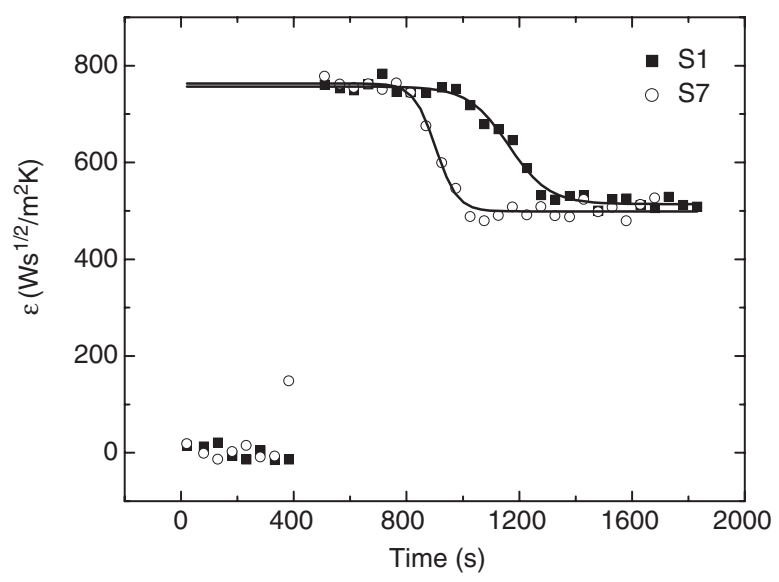

Figure 2. Variation of the thermal effusivity as a function of time for S1 and S7 samples. The square filled points are experimental values for S1 sample, the hollow circle points correspond to S7 sample and the continuous lines are the best data fitting of equation (4). where $\varepsilon_{\mathrm{e}}$ and $\varepsilon_{\mathrm{p}}$ are the thermal effusivities of the emulsion and the polystyrene latex film respectively, $t_{\mathrm{d}}$ is related to the time interval where the maximum variation in the effusivity occurs and $t_{0}$ is identified as the time at which the concavity of the sigmoid curve changes.

Fitting equation (4) to the experimental data, it is found that the thermal effusivities for the emulsion and cured polystyrene as a function of the manufacturing process have constant values (the mean values are $810 \mathrm{~W} \mathrm{~s}^{1 / 2} \mathrm{~m}^{-2} \mathrm{~K}^{-1}$ and $528 \mathrm{~W} \mathrm{~s}^{1 / 2} \mathrm{~m}^{-2} \mathrm{~K}^{-1}$, respectively). These results are explained taking into account the fact that, during the manufacturing process, the different emulsions have approximately the same concentration of polystyrene.

The characteristic curing time interval is identified with the parameter $2 t_{\mathrm{d}}$. As can be observed in figure 3, this parameter decreases from 162 to $78 \mathrm{~s}$ as a function of the manufacturing stage. Therefore, the maximum value of the curing velocity should increase, as it can be seen in figure 2 .

The times $t_{-}=t_{0}-t_{\mathrm{d}}$ and $t_{+}=t_{0}+t_{\mathrm{d}}$ are identified as the times when the curing of a layer of one thermal length of thickness, adjacent to the substrate, begins and finishes, respectively.

In order to understand the results, it is important to remark that, in photothermal techniques, the most important contribution to the temperature changes of the material surface comes from the properties of a material layer with a thickness given by one thermal diffusion length. In this case, the thermal length changes in time and is a function of the structure and thickness of the layer system (emulsion and cured polystyrene film).

The thermal properties of an inhomogeneous material are complicated functions of its features, being affected by the composition, structure, interfaces and, in general, all kind of inhomogenities in the material. Diverse studies have been developed in order to understand this problem; however, even for simple systems, composed by few homogeneous layers, these problems are still under study [17-19].

In our case, the effective thermal diffusion length $\left(\mu_{\mathrm{effec}}\right)$ depends on both materials (emulsion plus cured film). Due to the fact that polystyrene is permeable to water vapour [14], the

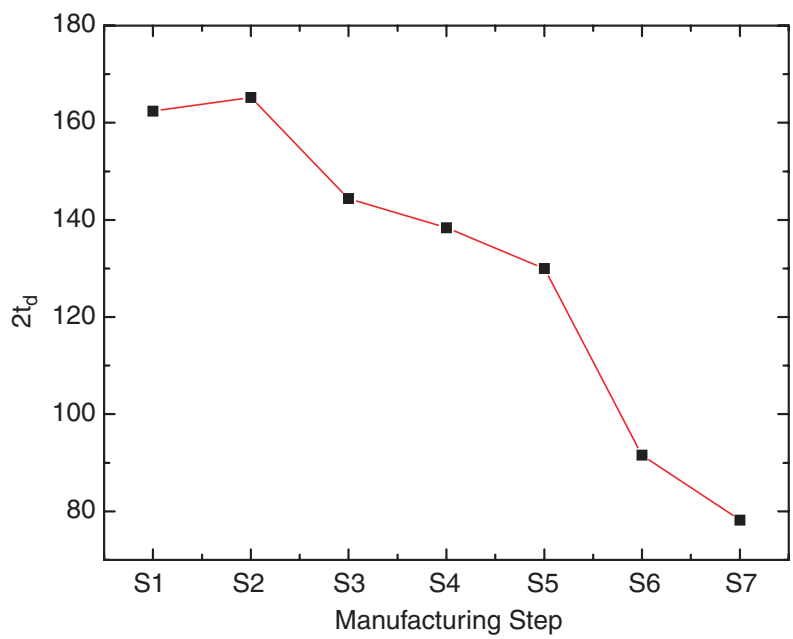

Figure 3. Characteristic curing time interval, $2 t_{\mathrm{d}}$, as a function of the manufacturing process. 
cure of the emulsion begins on the top (surface in contact with air) and finishes in the surface in contact with the reference material. With this in mind, it can be assumed that the system, latex film plus emulsion, is a homogeneous two-layer system that changes in thickness and in thermal properties as a function of time (see figure 4). In this figure, for the first seconds in the graph, $t<t_{-}$, the thermal properties detected by our PA technique configuration are those of the emulsion. For $t_{-}<t<t_{+}$, the thermal wave reaches both, the cured film and the emulsion which form the two-layer system mentioned above. For times $t_{+}<t$ the emulsion is cured completely and then the PA technique detects only properties of this material.

We applied, in this two-layer system, the in-series thermal resistor model [20] to obtain the effective thermal conductivity. The thermal resistance is defined by the ratio of the thickness to the thermal conductivity of the sample. In this approach, the effective resistance $R_{\text {effec }}$ of the two-layer system is given by

$$
R_{\text {effec }}=\frac{\mu_{\text {effec }}}{k_{\text {effec }}}=R_{\mathrm{p}}+R_{\mathrm{e}}=\frac{l_{\mathrm{p}}}{k_{\mathrm{p}}}+\frac{l_{\mathrm{e}}}{k_{\mathrm{e}}},
$$

here $l_{\mathrm{p}}(t)$ is the thickness of cured film, in the layer adjacent to the steel foil of total thickness $\mu_{\mathrm{effec}}(t)=l_{\mathrm{p}}(t)+l_{\mathrm{e}}(t)$, whose thermal properties are sensed by the PA detector, at any time $t$.

From equation (5) it is obtained that

$$
k_{\mathrm{effec}}=\frac{k_{\mathrm{e}}}{1+y(\lambda-1)},
$$

where $\lambda=k_{\mathrm{e}} / k_{\mathrm{p}}$, and $y$ is the fraction of emulsion cured at time $t$, given by

$$
y(t)=\frac{l_{\mathrm{p}}(t)}{\mu_{\mathrm{effec}}} .
$$

On the other hand, the effective heat capacity is

$$
(\rho c V)_{\mathrm{effec}}=(\rho c V)_{\mathrm{e}}+(\rho c V)_{\mathrm{p}},
$$

where $(\rho c)_{i}$ and $V_{i}$ are heat capacity per unit volume and volume, respectively, for $i=\mathrm{e}$ (emulsion) and $\mathrm{p}$ (cured polyestyrene).

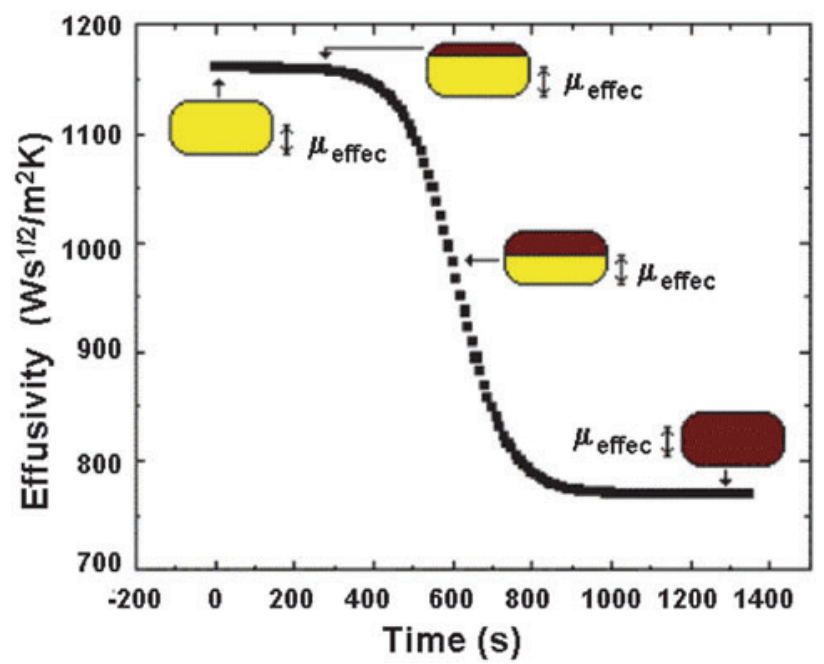

Figure 4. Photothermal model for the thermal effusivity measurements in inhomogeneous kinetic curing processes.
Taking into account that both layers have the same cross section, we obtain that the effective heat capacity per unit volume for the system is

$$
(\rho c)_{\mathrm{effec}}=(\rho c)_{\mathrm{e}}[1+y(\delta-1)],
$$

here $\delta=(\rho c)_{\mathrm{p}} /(\rho c)_{\mathrm{e}}$.

From equations (5), (8) and the relation among the thermal effusivity, conductivity and heat capacity per unit volume, the effective thermal effusivity is obtained:

$$
\varepsilon_{\mathrm{effec}}=\sqrt{(k \rho c)_{\mathrm{effec}}}=\varepsilon_{\mathrm{e}} \sqrt{\frac{1+y(\delta-1)}{1+y(\lambda-1)}} .
$$

Using equations (7), (10) and the relation for $\lambda$, we have that the cured film thickness detected, at time $t$, is given by

$l_{\mathrm{p}}(t)=\frac{\mu_{\mathrm{effec}}}{1-\left(k_{\mathrm{e}} / k_{\mathrm{p}}\right)\left[\left(\varepsilon_{\mathrm{p}}^{2}-\varepsilon_{\mathrm{effec}}^{2}(t)\right) /\left(\varepsilon_{\mathrm{e}}^{2}-\varepsilon_{\mathrm{effec}}^{2}(t)\right)\right]}$.

Derivating equation (11) in time, we obtain a 'velocity' $\left(v_{\mathrm{p}}(t)\right)$ of curing:

$$
v_{\mathrm{p}}(t)=\mu_{\mathrm{effec}} \frac{k_{\mathrm{e}}\left(\varepsilon_{\mathrm{p}}^{2}-\varepsilon_{\mathrm{e}}^{2}\right)}{\left[\left(k_{\mathrm{p}} \varepsilon_{\mathrm{e}}^{2}-k_{\mathrm{e}} \varepsilon_{\mathrm{p}}^{2}\right)+\left(k_{\mathrm{e}}-k_{\mathrm{p}}\right) \varepsilon_{\mathrm{effec}}^{2}(t)\right]^{2}} \frac{\mathrm{d}}{\mathrm{d} t}\left[\varepsilon_{\mathrm{effec}}^{2}(t)\right]
$$

and derivating once again, an 'acceleration' $\left(a_{\mathrm{p}}(t)\right)$ could be obtained:

$$
\begin{aligned}
a_{\mathrm{p}}(t)= & \mu_{\mathrm{effec}} k_{\mathrm{e}}\left(\varepsilon_{\mathrm{p}}^{2}-\varepsilon_{\mathrm{e}}^{2}\right)\left\{\left[\left(k_{\mathrm{p}} \varepsilon_{\mathrm{e}}^{2}-k_{\mathrm{e}} \varepsilon_{\mathrm{p}}^{2}\right)\right.\right. \\
& \left.+\left(k_{\mathrm{e}}-k_{\mathrm{p}}\right) \varepsilon_{\mathrm{effec}}^{2}(t)\right]\left(\mathrm{d}^{2}\left[\varepsilon_{\mathrm{effec}}^{2}(t)\right] / \mathrm{d} t^{2}\right) \\
& \left.-2\left(k_{\mathrm{e}}-k_{\mathrm{p}}\right)\left(\mathrm{d}\left[\varepsilon_{\mathrm{effec}}^{2}(t)\right] / \mathrm{d} t\right)^{2}\right\} \\
& \times\left[\left(k_{\mathrm{p}} \varepsilon_{\mathrm{e}}^{2}-k_{\mathrm{e}} \varepsilon_{\mathrm{p}}^{2}\right)+\left(k_{\mathrm{e}}-k_{\mathrm{p}}\right) \varepsilon_{\mathrm{effec}}^{2}(t)\right]^{-3} .
\end{aligned}
$$

In equations (11)-(13) there are three unknown magnitudes: $k_{\mathrm{p}}, k_{\mathrm{e}}$ and $\mu_{\mathrm{effec}}$. For $k_{\mathrm{p}}$, the value is reported in the literature [21], i.e. $k_{\mathrm{p}}=0.16 \mathrm{~W} \mathrm{~m}^{-1} \mathrm{~K}^{-1}$.

$k_{\mathrm{e}}$ can be obtained applying a random model [22], given by

$$
k_{\mathrm{e}}=k_{\mathrm{dp}}\left(\frac{k_{\mathrm{cp}}}{k_{\mathrm{dp}}}\right)^{x}
$$

where $k_{\mathrm{cp}}\left(0.61 \mathrm{~W} \mathrm{~m}^{-1} \mathrm{~K}^{-1}\right)$ and $k_{\mathrm{dp}}\left(0.16 \mathrm{~W} \mathrm{~m}^{-1} \mathrm{~K}^{-1}\right)$ are the thermal conductivities of the continuous (water) and disperse (polystyrene) [21] phase, respectively, and $x$ is the volume fraction of continuous phase in the emulsion, which was determined by weight loss (0.55). Substituting these values in equation (14) we obtain $k_{\mathrm{e}}=0.334 \mathrm{~W} \mathrm{~m}^{-1} \mathrm{~K}^{-1}$.

Due to the fact that we do not consider a model for $\mu_{\mathrm{effec}}$, which depends on time, we can only say that $l_{\mathrm{p}}(t)$ is bounded. The upper bound when $\mu_{\mathrm{effec}}=\mu_{\mathrm{e}}$ is considered and the lower bound when $\mu_{\mathrm{effec}}=\mu_{\mathrm{p}}$ is used. These thermal diffusion lengths are obtained from their relation with the thermal effusivity and conductivity, $\left(\mu_{i}=k_{i} /\left[(\pi f)^{1 / 2} \varepsilon_{i}\right]\right.$, $i=\mathrm{p}, \mathrm{e})$. For $\varepsilon_{i}$ we use the average thermal effusivity, obtained from the measurements of all the samples. Then, the values for the diffusion length for the cured film and of the not yet cured film are found to be $\mu_{\mathrm{p}}=45 \mu \mathrm{m}$ and $\mu_{\mathrm{e}}=61 \mu \mathrm{m}$.

The $\mu_{\text {effec }}$ value changes about $25 \%$ from its initial value $\mu_{\mathrm{e}}$ to the final value $\mu_{\mathrm{p}}$, so the error in the estimation of $l_{\mathrm{p}}$, according to equation (11), is also $25 \%$; then we choose the 


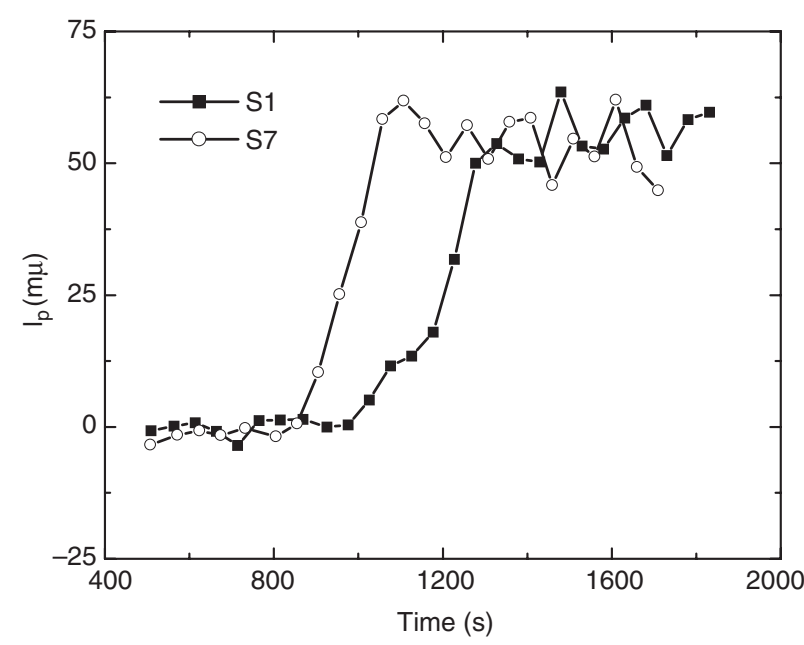

Figure 5. Thickness of cured polystyrene as a function of time for the S1 and S7 samples. The square filled points are experimental values for S1 sample and the hollow circle points correspond to S7 sample.

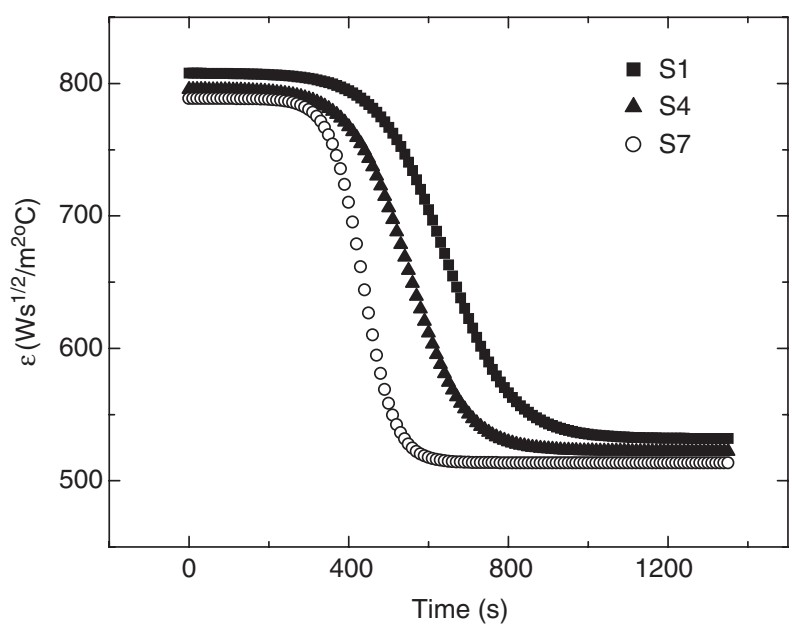

Figure 6. Thermal effusivity curves, for the samples S1, S4 and S7, obtained through equation (4) and the fitted parameters.

mean value to get a $12.5 \%$ variation. However, the general features of its time behaviour (curing time, velocity, etc) are not influenced by this assumption.

In figure 5, we show the thickness of cured polystyrene as a function of the time for S1 and S7 samples, using the value $\left(\mu_{\mathrm{e}}+\mu_{\mathrm{p}}\right) / 2$ as $\mu_{\mathrm{effec}}$. We can see that in the final stage of manufacturing, the curing process is faster than the first stage.

From now on, we will work with the parameters obtained from fitted curves to the data (from samples $51, \ldots, S 7$ ) and equation (4). In figure 6 the thermal effusivity curves for the samples S1, S4 and S7 are shown. In figures 7 and 8 , we show the velocities and accelerations for three representative samples, S1, S4 and S7. The maximum velocities and accelerations are shown in figures 9 and 10, respectively, for all the samples. We can see that these parameters increase in advanced stages of manufacturing.

It is worth mentioning that using this approach it is only necessary to monitor the thermal effusivity evolution in time of the sample in order to study the curing process.

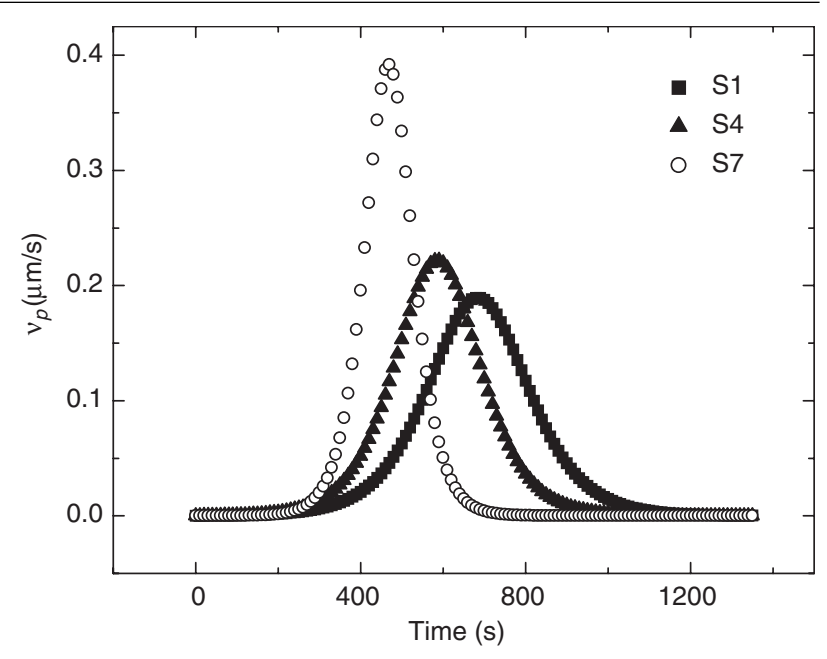

Figure 7. Velocities for the inhomogeneous kinetic curing process.

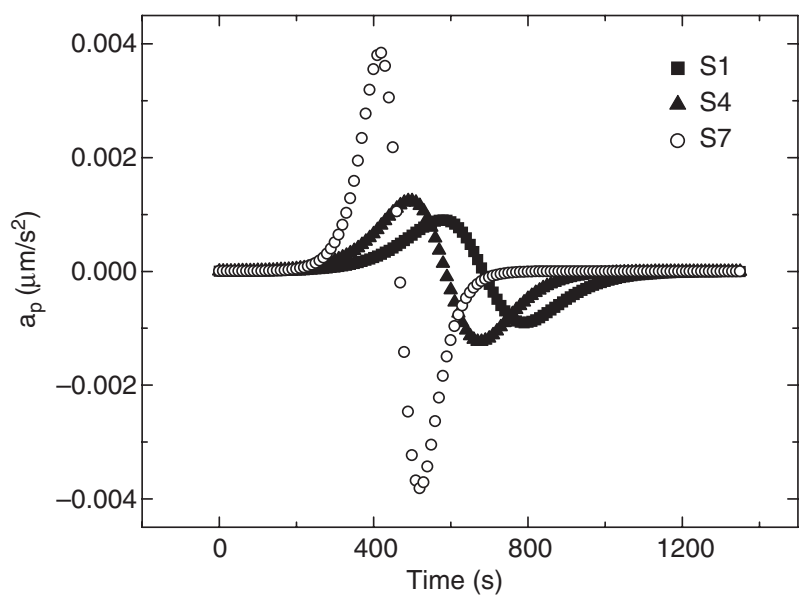

Figure 8. Accelerations for the inhomogeneous kinetic curing process.

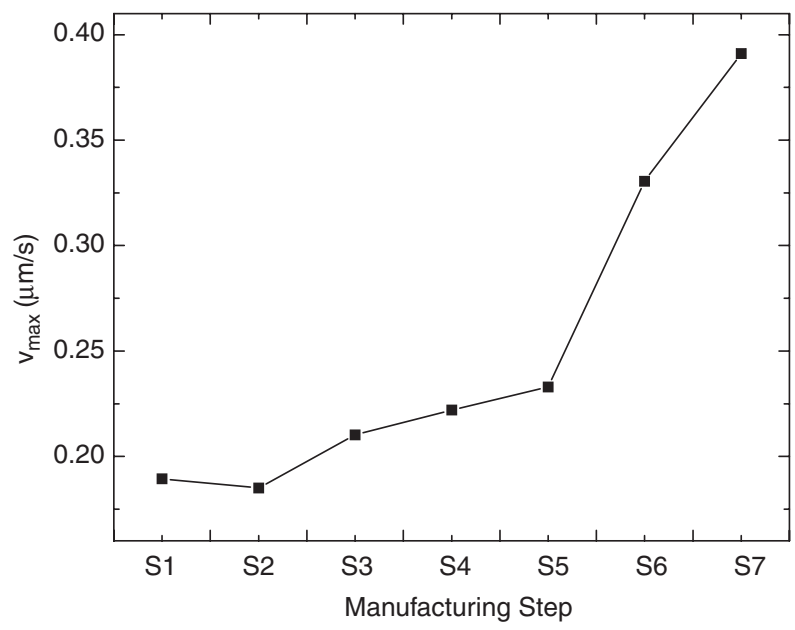

Figure 9. Maximum velocities as a function of the manufacturing steps.

\section{Conclusions}

It is shown that the time evolution of the inhomogeneous curing process of polystyrene emulsions can be studied using a variant 


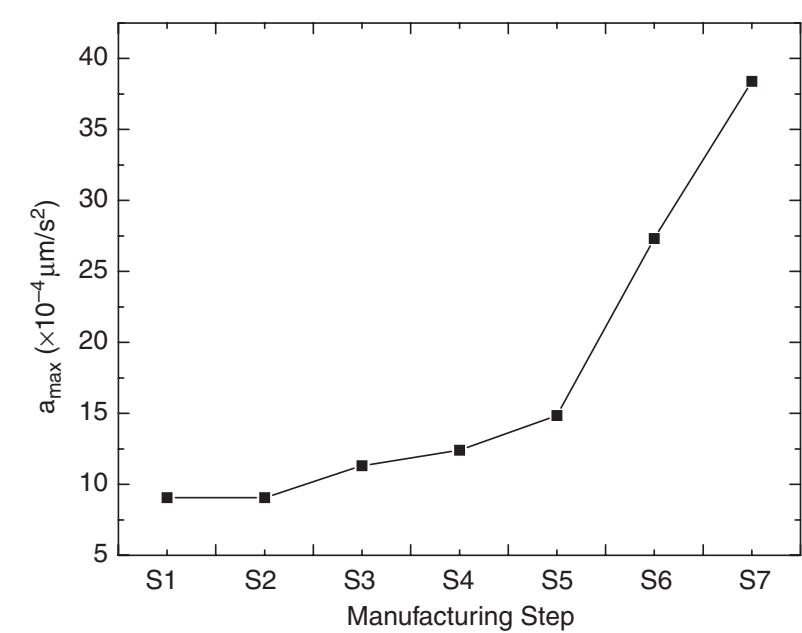

Figure 10. Maximum accelerations as a function of the manufacturing steps.

of the conventional PA technique. The thermal effusivity, as a function of time, was determined in order to monitor the curing process of a styrene emulsion in different steps of the manufacturing procedure. The behaviour of the thermal effusivity, as a function of time, shows a sigmoidal growth during the curing process. The parameterization of these curves permitted the determination of the characteristic curing time and velocity of the process. A decrease of the curing time and an increase of the curing velocity for the final steps of the manufacturing process were observed.

It has been shown that the PA experimental setup given here can be used for thermal effusivity measurements in samples with good thermal contact with substrate and these measurements can be correlated with the cured process in complex samples such as polymer compound, so these measurements give a quantitative kinetic curing analysis. These results provide an alternative technique for quality control in curing monitoring and measurement of optimal times in manufacturing process.

\section{Acknowledgments}

The authors thank Dr Agustin Salazar Hernandez for their comments and suggestions, Ing. M Muñoz, Tec. M Noriega and Tec. R Martínez for their technical assistance. The emulsion samples for this work were provided by 'Productos GLAV S.A. de C.V.' from Mexico. This work was partially supported by CONACyT through grant No 27603E, by CONCYTEG under grant No 00-16-CONCyTEG/CONACyT-075 and by 'Productos GLAV S.A. de C.V.'.

\section{References}

[1] Almond D P and Patel P M 1996 Photothermal Science and Techniques (London: Chapman and Hall)

Vargas H and Miranda L C M 1988 Phys. Rep. 16143

[2] Rosencwaig A 1990 Photoacoustic and Photoacoustic Spectroscopy (Florida: Krieger)

Shen Y, Lu Z and Lindber J 2000 Appl. Opt. 394007

Diebold G J and Westervelt P J 1988 J. Acoust. Soc. Am. 84 2245

Diebold G J and Cao Y N 1997 Opt. Eng. 36417

[3] Chirtoc M, Bicanic D, Chirtoc I, Lubbers M, Arnscheidt B and Pelzl J 1995 J. Mol. Structure 348469

[4] Hodgkinson J 1998 Photoacoustic and photothermal detection of trace contaminants in water PhD Thesis University of Southampton

[5] Balderas-López J A, Acosta-Avalos D, Alvarado-Gil J J, Zelaya-Angel O, Sánchez-Sinencio F, Falcony C, Cruz-Orea A and Vargas H 1995 Meas. Sci. Technol. 61163 Leite N F and Miranda L C M 1992 Rev. Sci. Instrum. 634398

[6] Hladik J (ed) 1990 Métrologie des Propriétés Thermophysiques de Matériaux (Paris: Masson)

[7] Almond D and Patel P (ed) 1996 Photothermal Science and Techniques (London: Chapman and Hall)

[8] Balderas-López J A, Gutiérrez-Juárez G, Fonseca-Jaime M and Sánchez-Sinencio F 1999 Rev Sci. Instrum. 702069

[9] Chen G and Yeung E 1998 Anal. Chem. 602258

[10] Sell J A, Heffelfinger D M, Ventzeg P L G and Gilgenbach R M 1991 J. Appl. Phys. 691330

[11] Malkin S and Puchenkov O 1997 Life and Earth Sciences, Progress in Photothermal and Photoacoustic Science and Technology (Bellingham, WA: SPIE Optical Engineering Press)

Acosta-Avalos D, Alvarado-Gil J J, Vargas H, Frías-Henández J, Olalde-Portugal V and Miranda L C M 1996 Plant Sci. 119183

[12] Miranda L C M and Cella N 1993 Phys. Rev. B 473896

[13] Melrose J R and Heyes D M 1993 J. Chem. Phys. 985873

[14] Odian G 1991 Principles of Polymerization 3rd edn (New York: Wiley)

[15] Salazar A and Sanchez-Lavega A 1994 Rev. Sci. Instrum. 65 2896

[16] Rosencwaig A and Gersho A 1976 J. Appl. Phys. 4764

[17] Pichardo J L, Alvarado-Gil J J, Cruz A, Mendoza J G and Torres G 2000 J. Appl. Phys. 877740

[18] Salazar A, Sánchez-Lavega A and Terrón J M 1998 J. Appl. Phys. 843031

[19] Lucio-M J L, Alvarado-Gil J J, Zelaya-Angel O and Vargas H 1995 Phys. Status Solidi a 150695

[20] Mansanares A M, Bento A C, Vargas H, Leite N F and Miranda L C M 1990 Phys. Rev. B 424477

Mansanares A M, Vargas H, Galembeck F, Buijs J and Bicanic D 1991 Appl. Phys. 707046

[21] Mark H F and Bikales N M 1989 Encyclopedia of Polymer Science and Engineering 2nd edn (New York: Wiley). Also in Styrene CEFIC (European Chemical Industry Council) Sector Group. http://www.cefic.be/sector/styrene/ guide9801/02.htm

[22] Maroulis Z B, Shah K K and Saravacos G D 1991 J. Food Sci. 56773 